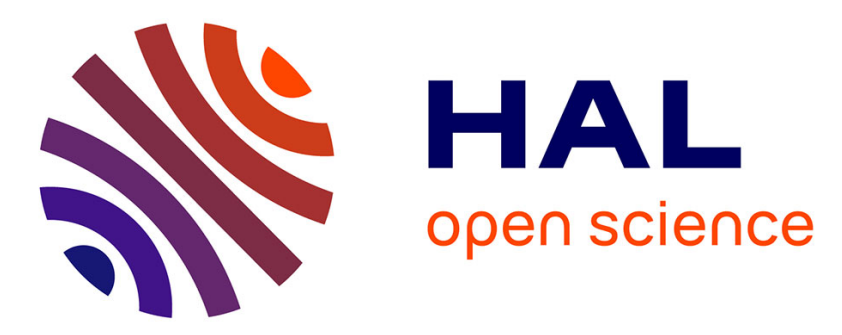

\title{
An automated optimization tool for high-dose-rate (HDR) prostate brachytherapy with divergent needle pattern
}

Maxence Borot de Battisti, M Maenhout, Baudouin Denis de Senneville, Gilion Hautvast, D Binnekamp, Jan Lagendijk, Marco van Vulpen, M Moerland

\section{To cite this version:}

Maxence Borot de Battisti, M Maenhout, Baudouin Denis de Senneville, Gilion Hautvast, D Binnekamp, et al.. An automated optimization tool for high-dose-rate (HDR) prostate brachytherapy with divergent needle pattern. Physics in Medicine and Biology, 2015, 60 (19), pp.7567-7583. hal01183579

\section{HAL Id: hal-01183579 \\ https://hal.science/hal-01183579}

Submitted on 10 Aug 2015

HAL is a multi-disciplinary open access archive for the deposit and dissemination of scientific research documents, whether they are published or not. The documents may come from teaching and research institutions in France or abroad, or from public or private research centers.
L'archive ouverte pluridisciplinaire HAL, est destinée au dépôt et à la diffusion de documents scientifiques de niveau recherche, publiés ou non, émanant des établissements d'enseignement et de recherche français ou étrangers, des laboratoires publics ou privés. 


\title{
An automated optimization tool for high-dose-rate (HDR) prostate brachytherapy with divergent needle pattern
}

\author{
M Borot de Battisti ${ }^{1}$, M Maenhout ${ }^{1}$, B Denis de Senneville ${ }^{2,3}$, \\ G Hautvast ${ }^{4}$, D Binnekamp ${ }^{4}$, J J W Lagendijk ${ }^{1}$, M van \\ Vulpen $^{1}$, M A Moerland ${ }^{1}$ \\ ${ }^{1}$ University Medical Center Utrecht, Dept. of Radiotherapy, Utrecht, The \\ Netherlands \\ ${ }^{2}$ University Medical Center Utrecht, Imaging Division, Utrecht, The Netherlands \\ ${ }^{3}$ IMB, UMR 5251 CNRS/University of Bordeaux, France \\ ${ }^{4}$ Philips Group Innovation - Biomedical Systems, Eindhoven, The Netherlands
}

E-mail: M.E.P.Borot@umcutrecht.nl

May 2015

\begin{abstract}
Focal High-Dose-Rate (HDR) for prostate cancer has gained an increasing interest as an alternative to whole gland therapy as it may contribute to reduction of treatment related toxicity. For focal treatment, optimal needle guidance and placement is warranted. This can be achieved under MRI guidance. However, MRI-guided needle placement is currently not possible due to space restrictions in the closed MR bore. To overcome this problem, a MR-compatible, single-divergent needle-implant robotic device is under development at the University Medical Centre, Utrecht (UMCU): placed between the legs of the patient inside the MR bore, this robot will tap the needle in a divergent pattern from a single rotation point into the tissue. This rotation point is just beneath the perineal skin to have access to the focal prostate tumor lesion. Currently, there is no treatment planning system commercially available which allows optimization of the dose distribution with such needle arrangement. The aim of this work is to develop an automatic inverse dose planning optimization tool for focal HDR prostate brachytherapy with needle insertions in a divergent configuration. A complete optimizer workflow is proposed which includes the determination of (1) the position of the center of rotation, (2) the needle angulations and (3) the dwell times. Unlike most currently used optimizers, no prior selection or adjustment of input parameters such as minimum or maximum dose or weight coefficients for treatment region and organs at risk is required. To test this optimizer, a planning study was performed on 10 patients (treatment volumes ranged from $8.5 \mathrm{~cm}^{3}$ to $23.3 \mathrm{~cm}^{3}$ ) by using 2 to 14 needle insertions. The total computation time of the optimizer workflow was below 20 minutes and a clinically acceptable plan was reached on average using only four needle insertions.
\end{abstract}




\section{Introduction}

Focal-HDR has gained an increasing interest as an alternative to whole-gland therapy in patients with localized prostate cancer. Focal therapy aims at reducing treatment related side effects and toxicity (Pieters et al 2009). The success of focal therapy will depend on the ability of tumour localization and dose delivery. Imaging, pathology and dose delivery studies have shown the value of multi-parametric MR-imaging for tumour localization (Groenendaal et al 2010). Furthermore, Polders et al (2015) showed an adequate dose coverage is obtained if a $5 \mathrm{~mm}$-margin is applied to the MR based tumour delineations. Therefore, a MRI-guided focal-HDR procedure is under investigation. In the daily practice at the UMCU, needles are inserted under ultrasound guidance while needle reconstruction, dose planning and needle positions verification are based on MRI. For optimal MR guidance during therapy, needle insertion should also be under MRI guidance, which is not currently possible due to access restrictions in a closed MR-bore system. To enable MR-guided needle insertion in the space restricted MR environment, MR-compatible robotic devices have been developed at several institutes: Fischer et al (2007 and 2008) and DiMaio et al (2007) recently designed robotic assistants for transperineal prostate needle placement. At the UMCU, a robotic device that can automatically insert needle into the patients prostate under MRI guidance is currently under development (Van den Bosch et al 2010). This robot is placed between the legs of the patients inside the MR bore. A tapping mechanism is used for needle insertion to restrict prostate movement and tissue deformation (Lagerburg et al 2006). Furthermore, needles are inserted under different angles in a divergent way, from a single rotation point. This rotation point is placed just beneath the perineal skin to have access to the whole gland. With this method, it is warranted to deliver the irradiation dose, needle per needle, guided by an adaptive planning system that takes anatomy changes and needle deviations into account. The ultimate goal is therefore to develop a procedure where the parameters of the dose plan are re-optimized after each needle insertion according to the perturbations of the set-up (change of anatomy, needle bending,...). For this purpose, a fast, accurate and stable optimization algorithm is important for inverse planning, enabling the implementation of dose-adaptive focal-HDR brachytherapy in the future. Currently, there is no commercial treatment planning system available which allows divergent needle insertion and adaptive planning. The development of such a treatment planning method is of great importance to find the most optimal implant in regard to planning target volume (PTV) coverage and sparing of organs at risk.

For the planning of brachytherapy intervention, various optimization methods have been developed. The most common algorithms deal with the dwell-times distribution within already implanted catheters such as geometrical optimization (GO) (KolkmanDeurloo et al 1994) or inverse planning simulated annealing (IPSA) (Lessard and Pouliot 2008, Alterovitz et al 2006). Some algorithms have been developed to determine the distribution of catheters within the prostate such as the 2D Centroidal Voronoi Tesselations (CVT) algorithm (Poulin et al 2013). This CVT algorithm optimizer has 
been described only for parallel needle configurations. Siauw et al (2012) described a needle planning by integer program (NPIP) algorithm to generate needle configurations that avoid critical structures near the penile bulb and other healthy structures, and avoid needle collisions inside the body. It was not applied for a divergent needle pattern with a single rotation point. More recently, hybrid algorithms which optimize the catheter positions and the dwell times have also been under investigation. Holm et al (2013) has developed a heuristics method for catheter positioning and dwell time distribution for parallel needle pattern with a runtime of $1 \mathrm{~h}$ without constraints regarding the nonperforation of urethra by the needles. Gorissen et al (2013) has developed a hybrid optimizer by using mixed integer programming with a runtime of several minutes.

All mentioned optimizers have certain drawbacks: First, most of them use parallel needle insertions and are not applicable for divergent needle insertion with a single point of insertion. Therefore, they cannot be used to determine the needle angulations or the position of the center of insertion. Second, they usually require numerous iterations and may produce sub-optimal dose results due to the trapping in local minima regions of the cost function landscape. Third, most optimizers require the manual determination of several input parameters as minima or maxima for PTV and organs at risk. In clinical practice however, dose coverage to the PTV and dose to the organs at risk are used to evaluate if the plan is clinically acceptable and therefore it is desirable to perform an optimization based on these parameters. Fourth, most optimizers usually require weight penalties (or importance coefficients) as input (see Alterovitz et al 2006 and Hsu et al 2004). These values depend on the patient anatomy. Therefore, weight penalties are not intuitive for a clinician and often need individual adjustment to obtain an acceptable plan. It is therefore necessary to generate a dose plan which will minimize the dose deposition error resulting from the weight penalty dependencies. Finally, in order to be eligible for intra-operative use, the total calculation time to obtain a clinically acceptable plan must be less than several minutes. This is usually not the case for most optimizers.

The aim of this article is to describe the development of a fully automatic inverse dose planning optimization tool for MRI-guided focal-HDR brachytherapy on prostate with a divergent needle pattern. Its goal is to determine the optimal center of rotation, needle angulations, source positions and dwell times within a reasonable time needed for intra-operative use. The optimizer will be tested in a planning study by assessing the dose volume parameters.

\section{Methods}

\subsection{Specification of the optimizer}

The coordinate system for the anatomy, the needle and the source positions is defined as follows. Let $\boldsymbol{r}=(x, y, z)$ be the spatial coordinate where $x, y$ and $z$ correspond to the positions in the left-right, the anteroposterior and the inferior-superior direction, respectively. For this divergent needle technique with the needle tracks coming from a 
single entry point, let $\boldsymbol{r}_{\text {rot }}=\left(x_{\text {rot }}, y_{\text {rot }}, z_{\text {rot }}\right)$ be the position of the center of rotation of the set-up and $\left(\theta_{i}, \phi_{i}\right)$ the angle of the $i^{\text {th }}$ needle insertion in the spherical coordinate system. In the common practice of HDR brachytherapy, the distance $\Delta$ between the dwell positions along the needle is constant. Due to the finite size of the needle, the number $N_{\text {source }}$ of possible dwell positions of the source along the needle is limited. The dwell positions $\boldsymbol{r}_{k}^{i}\left(\boldsymbol{r}_{\text {rot }}, \theta_{i}, \phi_{i}\right), k \in\left[1, N_{\text {source }}\right]$ of the $i^{\text {th }}$ needle insertion can then be expressed in Cartesian coordinates as follows:

$$
\boldsymbol{r}_{k}^{i}\left(\boldsymbol{r}_{r o t}, \theta_{i}, \phi_{i}\right)=\left[\begin{array}{l}
x_{r o t}+k \Delta \sin \left(\theta_{i}\right) \cos \left(\phi_{i}\right) \\
y_{r o t}+k \Delta \sin \left(\theta_{i}\right) \sin \left(\phi_{i}\right) \\
z_{r o t}+k \Delta \cos \left(\theta_{i}\right)
\end{array}\right]
$$

To develop a fully automatic optimizer for a given number of divergent needle insertions (referred to as $N_{\text {needle }}$ needles in the scope of this study), the following parameters need to be optimized: (1) the position of the center of rotation $\boldsymbol{r}_{\text {rot }}=$ $\left(x_{\text {rot }}, y_{\text {rot }}, z_{\text {rot }}\right) ;(2)$ the angles of the needle tracks $\left(\theta_{1 \cdots N_{\text {needle }}}, \phi_{1 \cdots N_{\text {needle }}}\right)$ in the spherical coordinate system; and (3) the dwell times of the sources $t_{k}^{i}$ at the source position $k$ $\left(k \in\left[1, N_{\text {source }}\right]\right)$ of the $i^{t h}$ needle insertion $\left(i \in\left[1, N_{\text {needle }}\right]\right)$.

Let $p=\left(\boldsymbol{r}_{\text {rot }}, \theta_{1 \cdots N_{\text {needle }}}, \phi_{1 \cdots N_{\text {needle }}}, t_{1 \cdots N_{\text {source }}}^{1}, \cdots, t_{1 \cdots N_{\text {source }}}^{N_{\text {needle }}}\right)$ be the vector containing the parameters of the set-up to be optimized and $\Omega$ its corresponding set of feasible solutions.

Finally, for a given dose plan, let $D_{95 \% \text { PTV }}, D_{10 \% \text { Ur }}, D_{1 c c \text { Rec }}$ and $D_{1 c c ~} B l$ be the dose received by $95 \%$ of the PTV, by $10 \%$ of the urethra and by $1 \mathrm{~cm}^{3}$ of rectum and bladder respectively. The optimizer must fulfill the following objectives: 1) highest coverage of the PTV (i.e. a large $D_{95 \%}$ PTV) and 2) dose on defined organs at risks (OAR) as low as possible (i.e. a small $D_{10 \% U r}, D_{1 c c} \operatorname{Rec}$ and $D_{1 c c B l}$ ).

The goal of the proposed optimizer is to determine the optimal set of parameters

$p_{\text {optimal }}=\left(\boldsymbol{r}_{\text {rot }}, \theta_{1 \cdots N_{\text {needle }}}, \phi_{1 \cdots N_{\text {needle }}}, t_{1 \cdots N_{\text {source }}}^{1}, \cdots, t_{1 \cdots N_{\text {source }}}^{N_{\text {needle }}}\right)_{\text {optimal }}$ to obtain the desired coverage in the PTV without exceeding the constraints of the organs at risk. Therefore, the following constraints function as input of the optimizer: $D_{95 \% \text { PTV }}^{\min }, D_{10 \% \text { Ur }}^{\max }, D_{1 c c}^{\max R e c}$ and $D_{1 c c B l}^{\max }$. They correspond to the minimum value of $D_{95 \% \text { PTV }}$ and the maximum value of $D_{10 \% U r}, D_{1 c c}$ Rec and $D_{1 c c} B l$ respectively in order to obtain a clinically acceptable and optimal dose plan. Concretely, a dose plan is clinically acceptable when: $D_{95 \% \text { PTV }}>D_{95 \% \text { PTV }}^{\min }, D_{10 \% \text { Ur }}<D_{10 \% \text { Ur }}^{\max }, D_{1 c c \text { Rec }}<D_{1 c c \text { Rec }}^{\max }$ and $D_{1 c c B l}<D_{1 c c B l}^{\max }$.

As output, the optimizer should give the optimal parameters of the set-up $p_{\text {optimal }}$ which corresponds to the maximum $D_{95 \%} P T V$ in combination with the minimization of $D_{10 \% \text { Ur }}, D_{1 c c \text { Rec }}$ and $D_{1 c c ~ B l}$.

\subsection{Dose computation and proposed optimization workflow}

According to the guideline of the AAPM Task group No. 43 (Nath et al 1995, Rivard et al 2004), the dose $D(\boldsymbol{r}, p)$ received at $\boldsymbol{r}$ is expressed as the sum of the contribution 


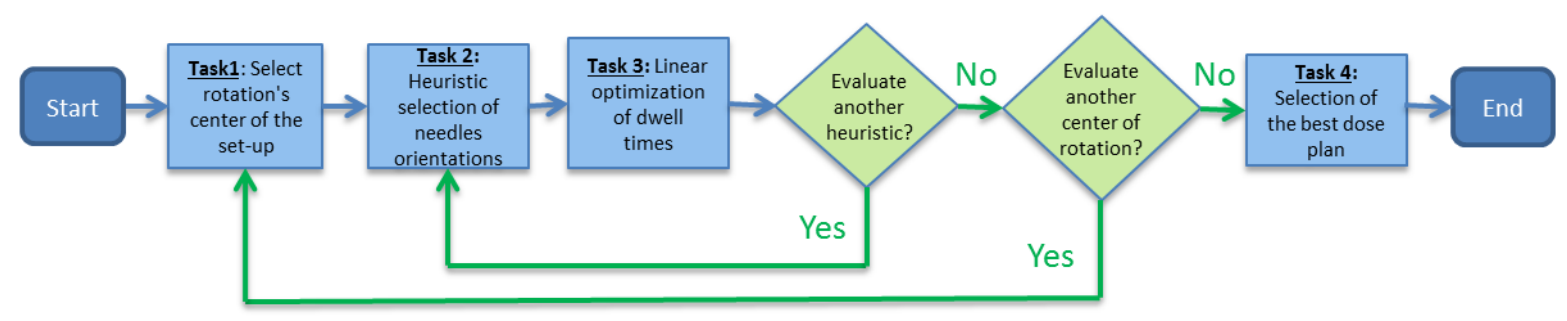

Figure 1. Complete optimization workflow for the determination for the position of the center of rotation, the needle orientations and the dwell times of the source.

of all source positions:

$$
D(\boldsymbol{r}, p)=\sum_{i=1}^{N_{\text {needle }}} \sum_{k=1}^{N_{\text {source }}} d_{k}^{i}\left(\boldsymbol{r}, \boldsymbol{r}_{\text {rot }}, \theta_{i}, \phi_{i}\right) t_{k}^{i}
$$

where $d_{k}^{i}\left(\boldsymbol{r}, \boldsymbol{r}_{\text {rot }}, \theta_{i}, \phi_{i}\right)$ is the dose-rate of source position $k$ of the $i^{t h}$ needle insertion and, depending on the model of the dose distribution chosen (point source, line source,...), is usually a complex non-linear function of $\boldsymbol{r}, \boldsymbol{r}_{\text {rot }}, \theta_{i}$ and $\phi_{i}$.

A way to obtain the desired dose plan is to approach the dose to a given value $D_{\text {opt }}(\boldsymbol{r})$ for all points $\boldsymbol{r}$ by solving the following equations:

$$
\begin{aligned}
& p_{\text {optimal }}=\underset{p \in \Omega}{\operatorname{argmin}}[C(p)] \\
& \text { with } C(p)=\iiint_{\boldsymbol{r} \in \mathbb{R}} \omega(\boldsymbol{r})\left[D(\boldsymbol{r}, p)-D_{\text {opt }}(\boldsymbol{r})\right]^{2} d \boldsymbol{r}
\end{aligned}
$$

where $C(p)$ is called the cost function, $p_{\text {optimal }}$ are the optimal parameters defined previously and $\omega(\boldsymbol{r})$ is the weight coefficient at the point $\boldsymbol{r}$ which will be detailed in section 2.5 .

The strategy of the proposed optimizer benefits from the linear impact of the dwell times on the deposited dose. If the center of rotation and the angles of the needle tracks are fixed, determining the value of $t_{k}^{i}$ goes back to solving a set of linear equations and it is thus feasible to find a direct and efficient solution. However, solving Eq. 5 for the variables of the needle positioning $\left(\boldsymbol{r}_{\text {rot }}, \theta_{1 \cdots N_{\text {needle }}}\right.$ and $\left.\phi_{1 \cdots N_{\text {needle }}}\right)$ is highly non-convex and consequently difficult to overcome. Therefore, the proposed method relies on the exact determination of the optimal dwell times using the resolution of linear equations while the remaining variables (position of center of rotation and angulations of needles) are deduced using heuristic or exhaustive searches. Since the area of insertion of the needle is reduced to several square centimeters in order of magnitude, an exhaustive enumeration of $\boldsymbol{r}_{\text {rot }}$ is employed. However, regarding the determination of the needle orientations $\left(\theta_{i}, \phi_{i}\right)$, an exhaustive optimization is highly time-consuming in practice. Therefore, the idea of evaluating a certain number of heuristics $N_{\text {heur }}$ for each center of rotation selected was employed to accomplish this task. The complete workflow of the optimizer is depicted in Figure 1. 

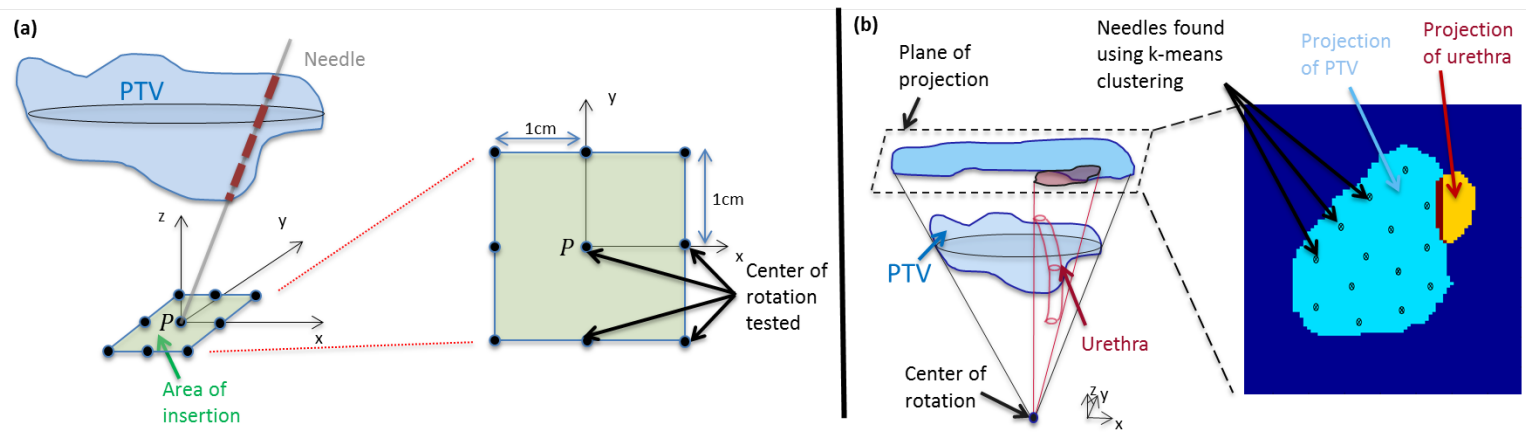

Figure 2. Figure 2(a) depicts the schematic representation of the exhaustive search of the position of the center of rotation. The area of insertion is represented in light green and the center of rotation evaluated are represented by the black dots. Figure 2(b) describes the proposed heuristics for the determination of needle orientations. First, the PTV and the urethra are projected from the center of rotation onto a transverse plane (cf. schematic on the left). A uniform distribution of needles is then found by applying $k$-means clustering on $P_{P T V}$ until no clusters are found in the urethra zone (cf. schematic on the right).

\subsection{Task 1: Exhaustive enumeration of the rotation center of the set-up}

The first task in the workflow is to select the position of the rotation center of the set-up (i.e. $\boldsymbol{r}_{\text {rot }}$ ).

The robotic device currently in development at the UMCU for HDR brachytherapy with divergent needle pattern is made such that the center of rotation should be placed at the perineum (to avoid multiple insertion points and to have full access to the prostate). Therefore, $\boldsymbol{r}_{r o t}$ is supposed fixed in the inferior-superior direction (i.e. $z$-axis) such that $z_{\text {rot }}$ is determined by the perineum. Furthermore, the ranges of robot movement are $2 \mathrm{~cm}$ along the $x$ and $y$-axis due to the restricted space between the legs. Thus, the values of $x_{\text {rot }}$ and $y_{\text {rot }}$ are initially chosen such that the point $P$ is in line with the center of the largest PTV contour in the transversal plane. An exhaustive search for the optimal center of rotation was performed by evaluating 9 possible candidates on a $1 \mathrm{~cm}$ grid (see Figure 2(a)).

\subsection{Task 2: Heuristic approach of the needle orientations}

At this point, the center of rotation is fixed. The next step is to construct several heuristics for the needle orientations. The heuristic approach proposed in this manuscript consists in finding a uniform needle distribution in space with the additional condition that none of the needle tracks perforates the urethra.

For this purpose, the method of Poulin et al (2013) for parallel needle set-up was adapted as follows: the PTV and urethra volume are projected from the center of rotation on a transverse plane behind the PTV (see Figure 2(a)). The latter projections are noted $P_{P T V}$ and $P_{U r e t h r a}$ respectively. The aim is to find a uniform distribution of $N_{\text {needle }}$ points in $P_{P T V}$ without any of those points inside $P_{\text {Urethra }}$. First, $N_{\text {needle }}$ initial 
generators are positioned randomly in $P_{P T V}$. Then, $k$-means clustering is applied on the indices of the surface $P_{P T V}$ with the additionnal condition that no cluster centers must stand in $P_{\text {Urethra }}$. The final cluster centers reflect the positions of the needle (see Figure 2(b)).

This method of heuristic selection relies on a random initial needle set up, therefore several heuristics were evaluated. The number of heuristics evaluated $N_{\text {heur }}$ in section 2.7.2.

\subsection{Task 3: Linear optimization of dwell times}

While entering this step, the center of rotation and the needle orientations are fixed. The determination of the dwell times can now be expressed using a linear optimization problem as follows.

In the following, $N_{\text {source }}^{\text {tot }}$ is the total number of active source positions and $t_{m}$ and $d_{m}(\boldsymbol{r})\left(m \in\left[1, N_{\text {source }}^{\text {tot }}\right]\right)$ are the dwell time and dose rate at each active source position respectively.

The final objective is a high PTV coverage while the OAR are spared as much possible. A way to reach this goal is to approach the dose to a certain value $D_{P T V}$ in the PTV and to 0 in the organs at risk. Considering a number $N_{O A R}$ organs at risk, the weight coefficients are supposed constant for the PTV and $O A R_{l}, l \in\left[1, N_{O A R}\right]$ and are noted $\omega_{P T V}$ and $\omega_{O A R_{l}}$ respectively in the following. In a discrete space, the cost functions of the PTV and the $O A R_{l}$ can be defined by referring to the definition of $C(p)$ in Eq. 3, as follows:

$$
\begin{aligned}
& C_{P T V}(p)=\frac{\omega_{P T V}}{V_{P T V}} \sum_{\boldsymbol{r} \in P T V}\left[D(\boldsymbol{r}, p)-D_{P T V}\right]^{2} \\
& \forall l \in\left[1, N_{O A R}\right], C_{O A R_{l}}(p)=\frac{\omega_{O A R_{l}}}{V_{O A R_{l}}} \sum_{\boldsymbol{r} \in O A R_{l}} D(\boldsymbol{r}, p)^{2}
\end{aligned}
$$

These cost functions were divided by the volume of the organs to avoid volume dependency in the optimization.

Therefore the basic cost function could be expressed as:

$$
C(p)=C_{P T V}(p)+\sum_{l=1}^{N_{O A R}} C_{O A R_{l}}(p)
$$

The optimal values of $\omega_{O A R_{l \cdots N} N_{O R}}, \omega_{P T V}$ and $D_{P T V}$ are obviously dependent of the anatomy of the patient. Therefore, in the following sections, the algorithm to minimize $C(p)$ for a given value of $\omega_{O A R_{l \cdots N} N_{O A}}, \omega_{P T V}$ and $D_{P T V}$ is presented (section 2.5.1). In section 2.5.2, the method to determine the optimal values of $\omega_{O A R_{l \cdots N} N_{O A R}}, \omega_{P T V}$ and $D_{P T V}$ according to the patients anatomy is described.

2.5.1. Solution using matrix inversion. In this section, a direct and fast method to determine the dwell times of the source without unrealistic negative values is described for fixed values of $\omega_{O A R_{l \cdots N} N_{O R}}, \omega_{P T V}$ and $D_{P T V}$. This problem of finding the optimal 
source dwell times for fixed needle positions is analogous to finding the optimal beam intensities in external beam radiotherapy: this reverts to finding a solution of the inverse problem which does not yield unphysical negative values. Therefore, a similar algorithm described by Goldman et al (2005 and 2009) in the case of Intensity Modulated Radiation Therapy (IMRT) known as Fast Inverse Dose Optimization (FIDO) is used. Goldman described a method to obtain a direct solution of the inverse problem that avoids negative beamlet weights. It involves reformulating the organs at risk cost functions $C_{O A R_{l}}(p)$ : in Eq. $5, D(\boldsymbol{r}, p)^{2}$ is replaced by $\sum_{m=1}^{N_{\text {source }}^{\text {tot }}}\left[d_{m}(\boldsymbol{r}) t_{m}\right]^{2}$. By modifying the organs at risk cost functions, $C_{O A R_{l}}(p)$ will not be null through destructive interference effects between dwell times and most of the unphysical negative solutions are therefore excluded. Furthermore, the optimization problem is reduced to a set of linear equations.

With this method, the optimal dwell times are obtained by the matrix inversion:

$$
T=\alpha^{-1} \beta
$$

with $T=\left(t_{m}\right)_{m \in\left[1, N_{\text {source }}^{\text {tot }}\right]}$ is the vector of $N_{\text {source }}^{\text {tot }}$ elements containing the dwell times at all active source positions. $\beta$ is also a vector of $N_{\text {source }}^{\text {tot }}$ elements and $\alpha$ is a $N_{\text {source }}^{\text {tot }} \times N_{\text {source }}^{\text {tot }}$ matrix defined respectively as follows:

$\forall m \in\left[1, N_{\text {source }}^{\text {tot }}\right], \beta_{m}=\frac{\omega_{P T V} D_{P T V}}{V_{P T V}} \sum_{r \in P T V} d_{m}(\boldsymbol{r})$

$\forall m, n \in\left[1, N_{\text {source }}^{\text {tot }}\right], \alpha_{m n}=\frac{\omega_{P T V}}{V_{P T V}} \sum_{\boldsymbol{r} \in P T V} d_{m}(\boldsymbol{r}) d_{n}(\boldsymbol{r})+\sum_{l=1}^{N_{O A R}} \frac{\omega_{O A R_{l}}}{V_{O A R_{l}}} \sum_{\boldsymbol{r} \in O A R_{l}} d_{m}(\boldsymbol{r}) d_{n}(\boldsymbol{r}) \delta_{m n}$

where $\delta_{m n}$ is the Kronecker delta function.

2.5.2. Exhaustive search of the weight coefficients $\left(\omega_{P T V}, \omega_{O A R_{1 \cdots N} \ldots A R}\right)$ and the dose approached for the PTV (DPTV). As the matrix inversion in Eq. 7 is little time consuming to execute, it is possible to obtain the overall results by an exhaustive search of $\omega_{O A R_{l \cdots N} N_{O A}}, \omega_{P T V}$ and $D_{P T V}$. Consequently, no individual adjustment of these parameters is needed to obtain an acceptable plan and the dwell times found will be optimal for any anatomy. The exhaustive optimization of $\omega_{O A R_{l \cdots N} N_{O R}}, \omega_{P T V}$ and $D_{P T V}$ will be investigated in section 2.7.1.

\subsection{Task 4: Selection criterion}

The exhaustive enumeration of Task 1 (center of rotation of set-up), the several heuristics generated in Task 2 (needle orientations) and the exhaustive search $\omega_{O A R_{l \cdots N} \text { OAR }}, \omega_{P T V}$ and $D_{P T V}$ in Task 3 (dwell times) will gives several dose plans. The objective is now to select the best plan. The following criterion is proposed: the best plan has the highest value of energy $E$ (in $G y$ ) defined as:

$$
E=\min (A, B, C, D)
$$


with the relative parameters:

$$
\begin{aligned}
& A=D_{95 \% P T V}-D_{95 \% P T V}^{\min } \\
& B=D_{10 \% \text { max }}^{\max }-D_{10 \% \text { Ur }} \\
& C=D_{1 c c \operatorname{Rec}}^{\max }-D_{1 c c R e c} \\
& D=D_{1 c c B l}^{\max }-D_{1 c c B l}
\end{aligned}
$$

The parameters $A, B, C$ and $D$ represent the relative difference between the dose coverage parameters and the clinical constraints (set as input) of the PTV, the urethra, the rectum and the bladder, respectively. The higher the value of $A$, the better the dose coverage of the PTV. Moreover, the clinical constraint at the PTV is achieved if, and only if $A>0$. Consequently, the minimum value over $A, B, C$ and $D$ in Eq. 10 corresponds to the maximal dose covering error with respect to the clinical constraints within the region that receives the "worst" dose deposition. E therefore represents the quality of the dose plan: the greater $E$ is, the better the dose plan becomes (the dose plan is clinically acceptable if, and only if $E>0$ ).

In conclusion, by maximizing $E$, the plan which has all parameters optimized (maximum $D_{95 \% \text { PTV }}$ in combination with minimization of $D_{10 \% \text { Ur }}, D_{1 c c \text { Rec }}$ and $D_{1 c c ~ B l}$ ) is selected.

\subsection{Experimental validation}

The different steps in the optimizer workflow were assessed before the complete optimizer workflow was validated. In the first experiment, the dwell time optimization (see section 2.5) was analyzed by assessing the automatic search of $\omega_{O A R_{l \cdots N} N_{O R}}, \omega_{P T V}$ and $D_{P T V}$. In the second experiment, regarding the determination of the needle angulations (see section 2.4), the required number $N_{\text {heur }}$ of heuristic to evaluate was analyzed. The last experiment consisted in testing the complete optimizer workflow in a planning study by assessing the dose volume parameters.

The experiments were performed in a retrospective evaluation using clinical data from 10 patients. The delineations of the prostate tumors and the OAR considered (urethra, bladder, rectum and rest of the tissues) were made on a $1 \mathrm{~mm}^{3}$ resolution MRI image by an experienced oncologist. The PTV volumes ranged from $8.5 \mathrm{~cm}^{3}$ to $23.3 \mathrm{~cm}^{3}$ with a median of $16.1 \mathrm{~cm}^{3}$. For all experiments, the clinical values as input were: $D_{95 \% \text { PTV }}^{\min }=19 G y, D_{10 \% \text { Ur }}^{\max }=21 G y, D_{1 c c \text { Rec }}^{\max }=12 G y$ and $D_{1 c c B l}^{\max }=12 G y$. Those are the clinical constraint values for single fraction HDR brachytherapy as monotherapy used at the UMCU. For all experiments, varying numbers of needle insertions (from 2 to 14) were tested.

Regarding the source position the common procedure at the UMCU was adopted: for each needle insertion, the active source center positions were separated by a step-size of $\Delta=2.5 \mathrm{~mm}$ and situated inside the PTV with an extra margin of $3 \mathrm{~mm}$.

For dose calculation, the dose rate was calculated using the point source approximation model due to the minimum time of computation, with a small adaptation 
as follows to avoid over-optimization of the dose close to the source:

$d_{k}^{i}\left(\boldsymbol{r}, \boldsymbol{r}_{r o t}, \theta_{i}, \phi_{i}\right)=$

$S_{K} \Lambda g_{P}\left[R_{k}^{i}\left(\boldsymbol{r}, \boldsymbol{r}_{r o t}, \theta_{i}, \phi_{i}\right)\right] \Phi_{a n}\left[R_{k}^{i}\left(\boldsymbol{r}, \boldsymbol{r}_{r o t}, \theta_{i}, \phi_{i}\right)\right] \frac{R_{0}{ }^{2}}{R_{k}^{i}\left(\boldsymbol{r}, \boldsymbol{r}_{r o t}, \theta_{i}, \phi_{i}\right)^{2}+\exp \left[-R_{k}^{i}\left(\boldsymbol{r}, \boldsymbol{r}_{r o t}, \theta_{i}, \phi_{i}\right)^{2}\right]}$

where $S_{K}$ is the air-kerma strength, $\Lambda$ the dose-rate constant in water, $\Phi_{a n}(R)$ the one-dimensional anisotropy factor, $R_{0}$ denotes the reference distance which is specified to be $10 \mathrm{~mm}, g_{P}(R)$ corresponds to the radial dose function in the case of point source approximation model and $R_{k}^{i}\left(\boldsymbol{r}, \boldsymbol{r}_{r o t}, \theta_{i}, \phi_{i}\right)$ is the distance (in millimeters) between the source position $\boldsymbol{r}_{k}^{i}\left(\boldsymbol{r}_{\text {rot }}, \theta_{i}, \phi_{i}\right)$ and $\boldsymbol{r}\left(R_{k}^{i}\left(\boldsymbol{r}, \boldsymbol{r}_{r o t}, \theta_{i}, \phi_{i}\right)=\left\|\boldsymbol{r}_{k}^{i}\left(\boldsymbol{r}_{\text {rot }}, \theta_{i}, \phi_{i}\right)-\boldsymbol{r}\right\|_{2}\right)$. With this adaptation of the point source model, the dose has an upper limit value close to the source, therefore it reduces the numeric instabilities for $R_{k}^{i}\left(\boldsymbol{r}, \boldsymbol{r}_{r o t}, \theta_{i}, \phi_{i}\right)$ approaching 0 .

TG43 constants, anisotropy factor and radial dose function for the microSelectronHDR (Elekta/Nucletron, Veenendaal, The Netherlands) 192-Iridium source were taken from a study of Daskalov et al (1998) $\left(\Lambda=1.108 c G y \cdot h^{-1} \cdot U^{-1}\right)$ and an arbitrary source strength $S_{K}=40.80 \mathrm{mGy} \cdot h^{-1} \cdot \mathrm{m}^{2}$ was chosen. The multiplication of the radial dose function $g_{P}(R)$ and the anisotropy factor $\Phi_{a n}(R)$ were approximated by a $2^{n d}$ order polynomial fit $\left(g_{P}(R) \cdot \Phi_{a n}(R)=a_{0}+a_{1} R+a_{2} R^{2}\right)$. The coefficients for the fit were $a_{0}=1.11, a_{1}=-3.30 \cdot 10^{-3}$ and $a_{2}=3.12 \cdot 10^{-6}$, where $R$ is in millimeters.

As well as an evaluation of dose to target and OAR, dose homogeneity and conformity were also investigated. The parameters homogeneity $\left(H I_{150 \%}\right.$ and $\left.H I_{200 \%}\right)$ and conformal index $(C O I N)$ are defined respectively as:

$$
\begin{aligned}
& H I_{i \%}=\frac{V_{100 \% \text { PTV }}-V_{i \% \text { PTV }}}{V_{100 \% \text { PTV }}} \text { with } i \in\{150,200\} \\
& C O I N=\frac{\left(V_{100 \% \text { PTV }}\right)^{2}}{V_{P T V} V_{100 \% \text { body }}}
\end{aligned}
$$

where $V_{i \%}$ PTV $\left(V_{100 \%}\right.$ body respectively) is the volume inside the PTV (the total volume respectively) that receive $i \%$ (100\% respectively) of the prescribed dose i.e. $D_{95 \% \text { PTV }}^{\text {min }}$ ). The $H I_{i \%}$ measures the volumes fraction that receives between $100 \%$ and $i \%(i \in$ $\{150,200\})$ of the prescribed dose and the COIN compares the reference dose coverage in the PTV with the total volume in the reference isodose volume.

2.7.1. Assessment of the exhaustive search of $\omega_{P T V}, \omega_{O A R_{1} \cdots N_{O A R}}$ and $\left.D_{P T V}\right)$ for the dwell times optimization (Task 3). In this experiment, the automatic search of $\omega_{O A R_{l \cdots N} \ldots A R}, \omega_{P T V}$ and $D_{P T V}$ is assessed. The center of rotation was fixed in line with the center of the largest PTV contour in the transversal plane and the mid-plane of the tumor. A heuristic for the needle orientations was determined as described in section 2.4. The dwell times for all source positions were calculated using Eq. 7 for all possible combinations of weights coefficients of PTV, urethra, bladder, rectum and 
other tissues (respectively $\omega_{P T V}, \omega_{U r}, \omega_{B l}, \omega_{R e c}$ and $\omega_{\text {tissue }}$ ) and $D_{P T V}$ such that:

$$
\begin{aligned}
& D_{P T V} \in\{20,21, \cdots, 79,80\} \\
& \log _{10}\left(\omega_{P T V}\right) \in\{0,0.001, \cdots, 9.999,10\} \\
& \left(\log _{10}\left(\omega_{U r}\right), \log _{10}\left(\omega_{B l}\right), \log _{10}\left(\omega_{R e c}\right), \log _{10}\left(\omega_{\text {tissue }}\right)\right) \in\{0,1, \cdots, 9,10\}^{4}
\end{aligned}
$$

with the additional condition that:

$$
\log _{10}\left(\omega_{P T V}\right)+\log _{10}\left(\omega_{U r}\right)+\log _{10}\left(\omega_{B l}\right)+\log _{10}\left(\omega_{R e c}\right)+\log _{10}\left(\omega_{\text {tissue }}\right)=10
$$

to avoid redundancy of results.

From all resulting dose plans, the best one was selected with the selection criterion defined in section 2.6

In the following experiments (described in section 2.7.2 and 2.7.3), to obtain the optimal dwell time, the optimal values of $\omega_{P T V}, \omega_{U r}, \omega_{B l}, \omega_{R e c}$ and $\omega_{\text {tissue }}$ and $D_{P T V}$ were determined by applying the same exhaustive search. However, in order to decrease the time of calculation, the exhaustive search range of $\omega_{P T V}$ was reduced to:

$$
\log _{10}\left(\omega_{P T V}\right) \in\{0,1, \cdots, 9,10\}
$$

2.7.2. Analysis of the required number of heuristic for the needle angulations to be evaluated in Task 2. In this experiment, the number of heuristic evaluated $N_{\text {heur }}$ in Task 2 was assessed. The complete optimizer workflow was performed several times without an exhaustive search of the center of rotation (which was fixed in line with the center of the largest PTV contour in the transversal plane and the mid-plane of the tumor) for $N_{\text {heur }}$ varying from 1 to 80 . The energy, $E$, of the final dose plan was determined as a function of $N_{\text {heur }}$.

For the following experiment described in section $2.7 .3, N_{\text {heur }}$ was fixed to 10 to limit the time of calculation.

2.7.3. Analysis of the number of inserted needles. In this experiment, the optimizer was tested in a planning study by assessing the dose volume parameters. $p_{\text {optimal }}$ was determined to obtain the desired coverage without exceeding the constraints of the organs at risk for 2, 4, 6, 8, 10, 12 and 14 needle insertions by using the earlier mentioned constraints as inputs.

\section{Results}

In this section, the results of the experiment described in section 2.7 are presented below. Firstly, the results of the exhaustive search of $\omega_{P T V}, \omega_{U r}, \omega_{B l}, \omega_{R e c}$ and $\omega_{\text {tissue }}$ and $D_{P T V}$ to obtain optimal dwell times are described in section 3.1. Regarding the optimization of the needle angulations, the results of the analysis of the required number $N_{\text {heur }}$ of heuristic evaluations are described in section 3.2. Finally, the results of the planning study for one typical patient and afterwards, for all patients are shown in section 3.3. 


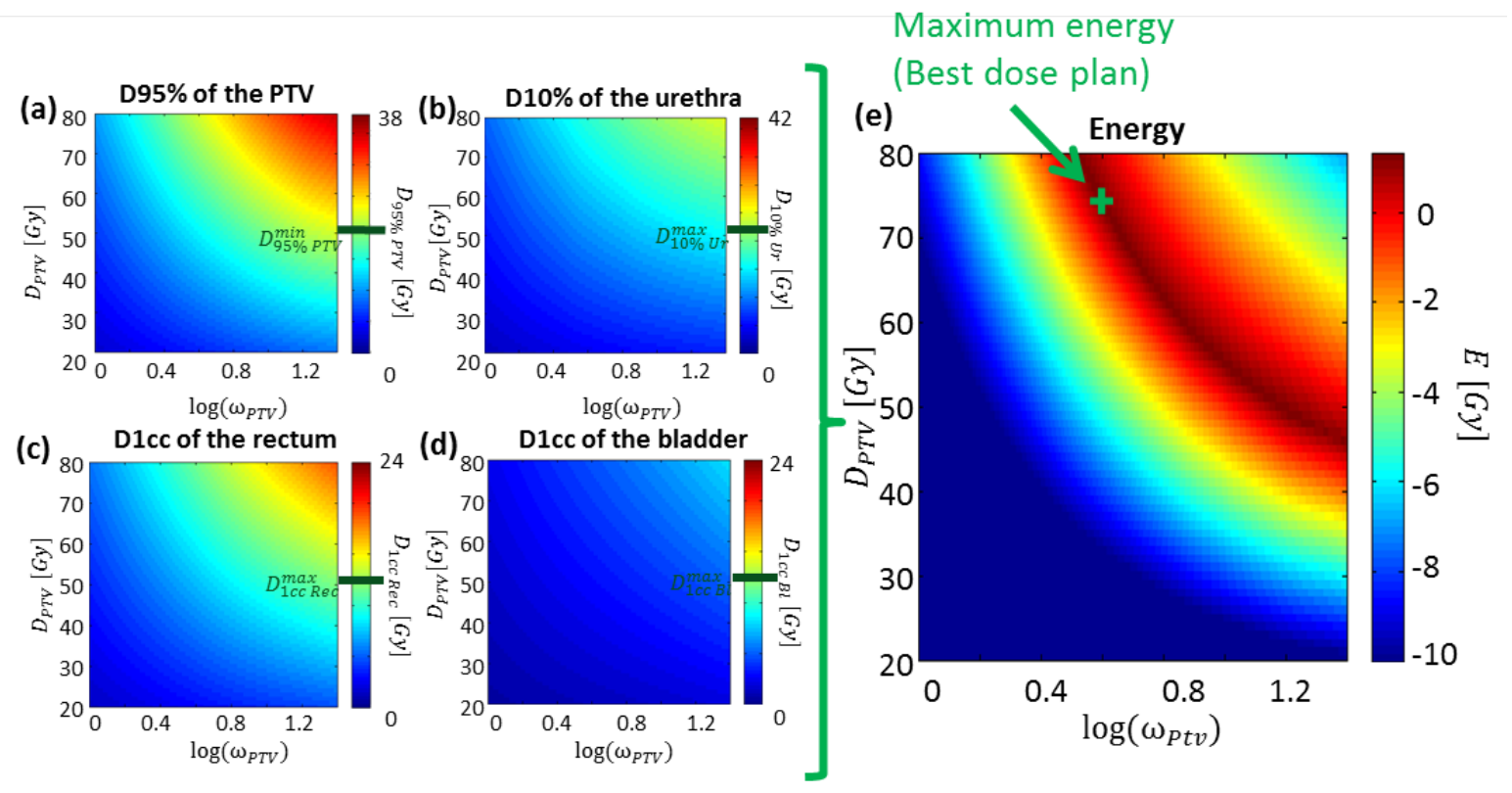

Figure 3. Results of the simulation obtained on a typical patient with 10 needle insertions. The values of $D_{95 \%}$ PTV $, D_{10 \% U r}, D_{1 c c} R e c, D_{1 c c} B l$ and $E$ (computed from Eq. 10) are presented as function of $\log _{10}\left(\omega_{P T V}\right)$ and $D_{P T V}$ (Figure 3(a), 3(b), $3(\mathrm{c}), 3(\mathrm{~d})$ and $3(\mathrm{e})$ respectively). $\log _{10}\left(\omega_{\text {tissue }}\right)$ was deducted from Eq. 17. On figure $3(\mathrm{a}), 3(\mathrm{~b}), 3(\mathrm{c})$ and $3(\mathrm{~d})$, the horizontal green lines represent the clinical constraints set as input.

\subsection{Assessment of the exhaustive search of $\omega_{P T V}, \omega_{O A R_{1 \cdots N_{O A R}}}$ and $\left.D_{P T V}\right)$ for the dwell times optimization (Task 3)}

In this section, the results of the experiment described in section 2.7.1 are presented in Figure 3 for one patient with 10 needle insertions as a typical example.

The best dose plan (i.e. the one maximizing $E$ ) was found with the following parameters: $\quad\left\{D_{P T V}, \omega_{P T V}, \omega_{U r}, \omega_{B l}, \omega_{R e c}, \omega_{\text {tissue }}\right\}=\left\{75,10^{0.587}, 10^{2}, 10^{4}, 10^{2}, 10^{1.413}\right\}$. The values of $D_{95 \%}$ PTV $, D_{10 \% U r}, D_{1 c c \text { Rec }}, D_{1 c c B l}$ and $E$ are plotted in Figure 3 as a function of $D_{P T V}$ and $\log _{10}\left(\omega_{P T V}\right)$. For the representation, $\left\{\omega_{U r}, \omega_{B l}, \omega_{R e c}\right\}$ were set to their optimal values $\left\{10^{2}, 10^{4}, 10^{2}\right\}$ and $\omega_{\text {tissue }}$ was deducted from Eq. 17.

Figure 3(a), 3(b), 3(c) and 3(d) show an increase of the dose deposition in all the different volumes considered (PTV, urethra, bladder and rectum) for increasing $D_{P T V}$ and $\log _{10}\left(\omega_{P T V}\right)$. In Figure 3(e), the best solutions (where $E$ is maximal) are disposed on a continuous line.

For $\log _{10}\left(\omega_{P T V}\right)>1.42$, one or more dwell times found with FIDO had negative values. These nonphysical solutions are due to the resolution of the equation: by modifying the organ at risk cost functions in FIDO, most of the solutions which give negative dwell times are removed but not all of them. 


\subsection{Analysis of the required number of heuristic for the needle angulations to be evaluated in Task 2}

In this section, the results of the experiment described in section 2.7.2 are presented in a situation of a large number of needle insertions $\left(N_{\text {needle }}=14\right)$ : This corresponds to the worst case scenario where the calculations are the most timeconsuming. For each patient, the energy $E$ of the selected dose plan was calculated within the number of heuristics evaluated. The average energy $\bar{E}$ on all 10 patients was plotted as a function of $N_{\text {heur }}$ (Figure 4 and the calculation time of the complete optimization workflow was also represented.

Figure 4 shows a fast initial increase of $\bar{E}$ with the number of evaluated heuristics. Afterwards, the gradient decreases progressively. For $N_{\text {heur }}=10$, $\bar{E}$ achieves $64 \%$ of its value for $N_{\text {heur }}=$

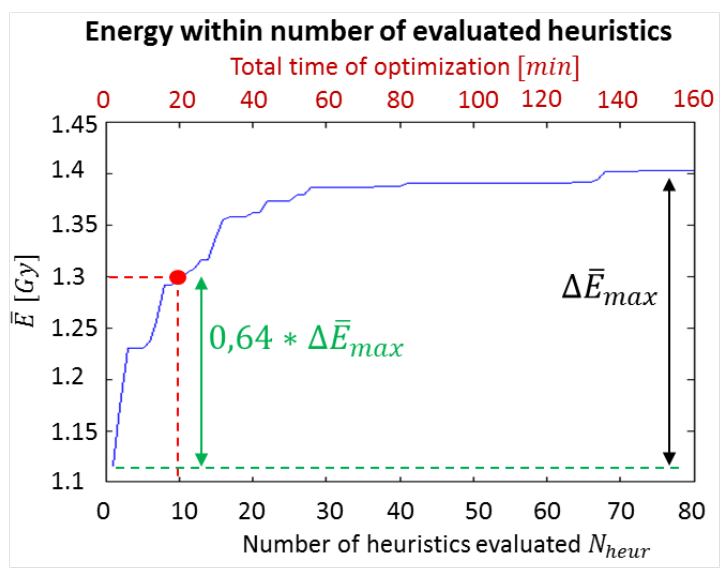
80. Furthermore, the number of heuristics evaluated in Task 2 is directly proportional to the calculation time of the complete optimization workflow. The time of optimization for $N_{\text {heur }}=10$ was approximatly 20 minutes on a PC with a $3.10 \mathrm{GHz}$ Intel@CoreTM i5-2400 processor and 8GB RAM using MATLAB R2013a.

\subsection{Analysis of the number of inserted needles}

The dose distribution was calculated on 10 patients (PTV volumes ranged from ranged from $8.5 \mathrm{~cm}^{3}$ to $23.3 \mathrm{~cm}^{3}$ with a median of $16.1 \mathrm{~cm}^{3}$ ) for $2,4,6,8,10,12$ and 14 needle insertions by using the earlier mentioned constraints as input. For each case, $D_{95 \%} P T V$, $D_{10 \% \text { Ur }}, D_{1 c c \text { Rec }}, D_{1 c c ~ B l}, H I_{150 \%}, H I_{200 \%}$ and $C O I N$ was computed.

The result for one typical patient is shown in Figure $5(\mathrm{a})(\mathrm{b})(\mathrm{c})$ and (d). The dose constraints set as input are also represented. $D_{95 \%}$ PTV increased with the number of needle insertions, and $D_{1 c c} \operatorname{Rec}$ and $D_{1 c c B l}$ decreased. Furthermore, for this patient,

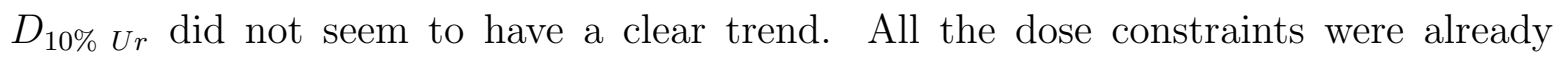
reached at four needle insertions. Moreover, $H_{200 \%}$ and $C O I N$ increased with the number of needle insertions, but the influence of $N_{\text {needle }}$ on $H I_{150 \%}$ was relatively small.

Figure 5(c) shows the MRI image of a sagittal plane of the same patient with delineations and figure $5(\mathrm{~d})$ and $5(\mathrm{e})$ show the dose distribution in this same sagittal plane for 2 and 10 needle insertions respectively. The cold spots in the PTV and the hot 

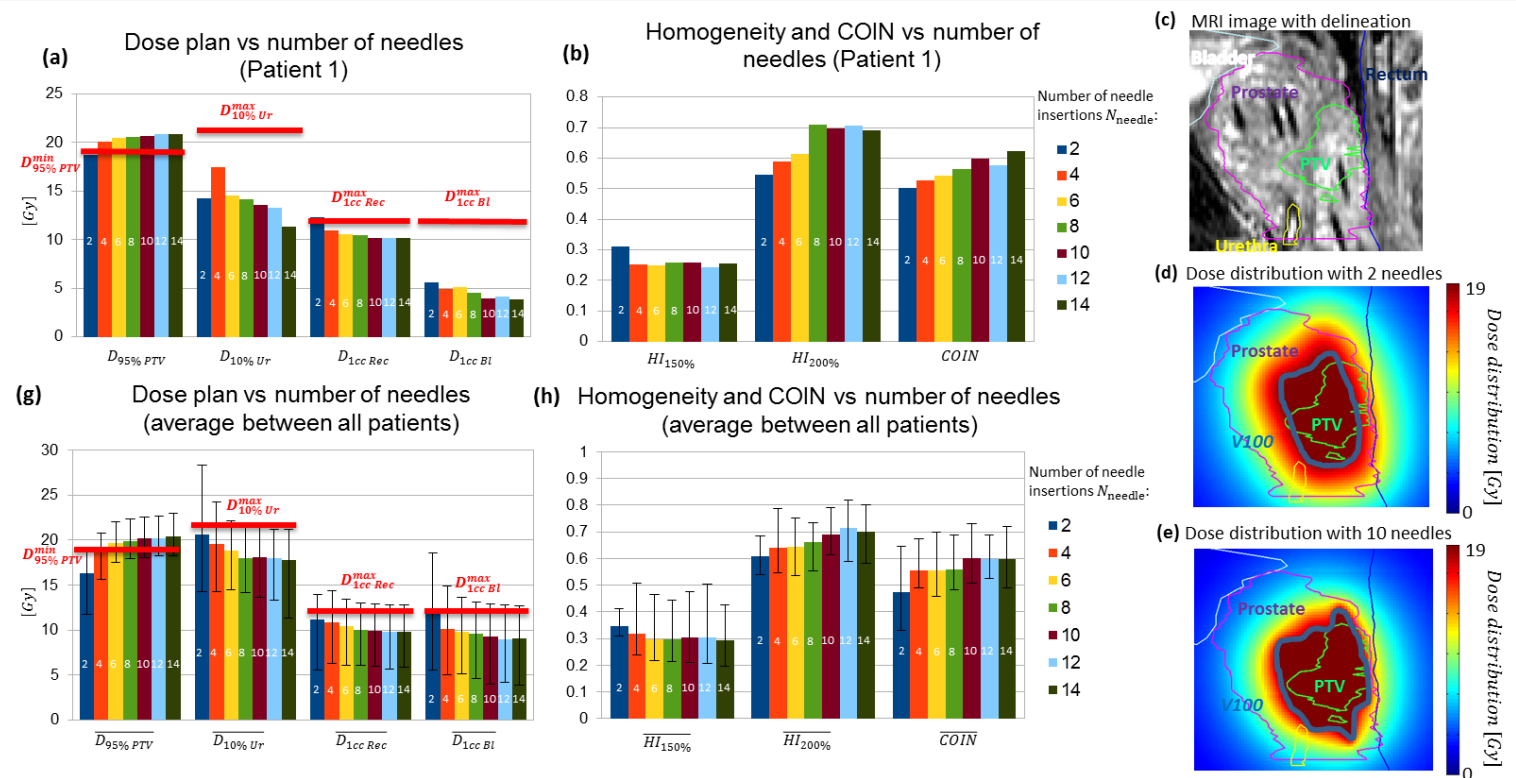

Figure 5. Results of the planning study for using 2, 4, 6, 8, 10, 12 and 14 needle insertions. Figure $5(\mathrm{a})(\mathrm{b})(\mathrm{c})(\mathrm{d})$ and (e) present the optimization results for a typical patient case. Figure $5(\mathrm{a})$ shows the output parameters $\left(D_{95 \%}\right.$ PTV $, D_{10 \% \text { Ur }}, D_{1 c c} \operatorname{Rec}$ and $\left.D_{1 c c B l}\right)$. Figure $5(\mathrm{~b})$ shows the Homogeneity $\left(H I_{150 \%}\right.$ and $\left.H I_{200 \%}\right)$ and the conformal index $(C O I N)$. Figure $5(\mathrm{c})$ presents a slice of MRI image in the sagittal plane with the delineations of the volumes of interest. Figures 5(d) and (e) present the corresponding dose distributions for 2 and 10 needle insertions respectively on the same sagittal slice. Figure 5(f) and (g) present the optimization results for 10 different patients. Figure $5(\mathrm{f})$ shows, the average output parameters on all patients $\overline{D_{95 \% ~ P T V}}$, $\overline{D_{10 \% U r}}, \overline{D_{1 c c \text { Rec }}}$ and $\overline{D_{1 c c B l}}$. Figure $5(\mathrm{~g})$ represents the average on all patients of homogeneity and conformal index $\left(\overline{H I_{150 \%}}, \overline{H I_{200 \%}}\right.$ and $\overline{C O I N}$ respectively). For the two latter graphs, the whole range of values is represented by the solid vertical line. For Figure 5(a)(b)(f) and (g), The red solid lines represent the clinical constraints set as input $\left(D_{95 \%}^{\min P T V}, D_{10 \% U r}^{\max }, D_{1 c c R e c}^{\max }\right.$ and $D_{1 c c B l}^{\max }$ respectively)

spots in the rectum were significantly reduced for $N_{\text {needle }}=10$ compared to $N_{\text {needle }}=2$.

For 2, 4, 6, 8, 10, 12 and 14 needle insertions the averaged parameters on all patients $\overline{D_{95 \% \text { PTV }}}, \overline{D_{10 \% \text { Ur }}}, \overline{D_{1 c c R e c}}, \overline{D_{1 c c ~ B l}}, \overline{H I_{150 \%}}, \overline{H I_{200 \%}}$ and $\overline{C O I N}$ are presented in Figure 5(g) and (h).

Once again, $\overline{D_{95 \%} P T V}$ increased on average with the number of needle insertions, while $\overline{D_{10 \% \text { Ur }}}, \overline{D_{1 c c R e c}}$ and $\overline{D_{1 c c ~ B l}}$. The large ranges of values were due to the different anatomies of the 10 patients tested. $\overline{H I_{200 \%}}$ and $\overline{C O I N}$ also increased with the number of needle insertions but $\overline{H I_{150 \%}}$ did not show any clear trend. On average, a clinical acceptable plan is already reached by using four needle insertions.

\section{Discussion}

In this manuscript, an automatic inverse dose planning optimization tool for MRIguided focal-HDR brachytherapy on prostate with divergent needle pattern is proposed. 
The aim was to determine the optimal parameters of the set-up (point of rotation, needles angles and dwell times) which corresponds to the maximization of $D_{95 \%} P T V$ in combination with minimization of $D_{10 \% U r}, D_{1 c c}$ Rec and $D_{1 c c} B l$. For that, the linear impact of the dwell times on the deposited dose was exploited and the remaining variables were determined by evaluating several heuristics for the needle angulations and by an exhaustive search for the position of the point of rotation.

Unlike most optimizers such as HIPO or IPSA, the proposed method does not require individual adjustments of several input parameters such as minimum dose, maximum dose or weight coefficients for PTV and organs at risk to obtain an acceptable plan (Dinkla et al 2014). Figure 3 illustrates the importance of an exhaustive optimization of the weight coefficients and approached dose at the PTV to determine the optimal dwell times. It shows that the dose deposition is made at the expense of all the other regions. In particular for this patient, the rectum played a very important role in the dose deposition. However, the dose deposition is a little less critical for the urethra and has little impact for the bladder. Thus, it is difficult to predict how the weight coefficients influence the overall dose plan. As an example, increasing $\omega_{P T V}$ will certainly increase $D_{95 \%}$ PTV , but its influence on $D_{10 \% \text { Ur }}, D_{1 c c}$ Rec or $D_{1 c c} B l$ is unknown: it could be insignificant or dramatic depending on the anatomy of the patient. However, the gain in energy would not significantly drop by using a larger step in the exhaustive search of $\log _{10}\left(\omega_{P T V}\right)$. Therefore, $\log _{10}\left(\omega_{P T V}\right)$ is varied from 0 to 10 with a step of 1 in order to reduce the time of optimization and still obtain a good dose plan.

An important step in brachytherapy and thus in the optimizer workflow is to find the optimal needle positions. The algorithms developed recently are usually based on iterative methods (Holm et al 2013, Gorissen et al 2013 and Siauw et al 2012) which cause two problems. First, such algorithms strongly depend on the initialization and therefore could produce sub-optimal solutions due to the trapping in local minima regions of the cost function landscape. The second problem is that, due to the high non-convexity of the problem, the optimization may require a long calculation time. To avoid these problems, a different approach was chosen: The angulation of the needle tracks is determined by evaluating several heuristics chosen carefully using the k-means clustering. According to Figure 4, for a given point of rotation, evaluating 10 heuristics already gives a good dose plan compared to 80 heuristics and a significantly better dose plan compared to one heuristic. By evaluating 10 heuristics, the total time of the optimization for a given number of needle insertions is below 20min. It is important to note that the program has been developed on Matlab and has not been optimized for speed. The proposed pipeline and the employed numerical schemes thus show great perspectives for a further reduction of the computation time using Graphical Processing Units (GPU's) in a parallel architecture. This will be studied in future work.

Finally, the planning study of the proposed optimizer workflow shows promising results. The clinical constraints set as input were reached on average with 4 needle insertions which is better compared to the current clinical procedure where 13 to 17 needles are usually inserted (see Hsu et al 2004, Menard et al 2004). More precisely, 
the increase of $D_{95 \%}$ PTV as well as the decrease of $D_{1 c c} \operatorname{Rec}$ or $D_{1 c c} B l$ with the number of needle insertions for the patient shown in Figure $5(\mathrm{a})$ was expected. $D_{10 \%}$ Ur did not depict a trend with the number of needle insertions: it already fulfilled by far the constraints for the urethra set as input $\left(D_{10 \% U r}^{\max }=21 G y\right)$ and therefore it was not the parameter to be optimized in priority. Moreover, $H I_{200 \%}$ and $C O I N$, expressing the dose homogeneity and conformity respectively, increased with the number of needle insertions as expected. $H I_{150 \%}$ did not show a clear trend. In this study, the PTV volumes had a median of $16.1 \mathrm{~cm}^{3}$ and all the dose constraints were already reached at 4 needle insertions on average. Therefore, those results are in line with the study of Steggerda et al (2010) which shows, in the case of Low-Dose-Rate (LDR) brachytherapy, satisfactory dose coverage for, on average, 0.3 needles per $\mathrm{cm}^{3}$ prostate volume. Furthermore, Vargas et al (2004) and Boyea et al (2007) showed the urinary toxicity following HDR brachytherapy is significantly increased by using more than 14 needle insertions. The cold spot in the PTV for 2 needle insertions as presented on Figure 5(d) was reduced by an increasing number of needle insertions (see Figure 5(e)). This illustrates how the number of needle insertions could improve the dose distribution. Figures $5(\mathrm{~g})$ and $5(\mathrm{f})$ confirms the observation shown in Figures 5(a) and 5(b) for a typical patient. $\overline{D_{95 \% ~ P T V}}$

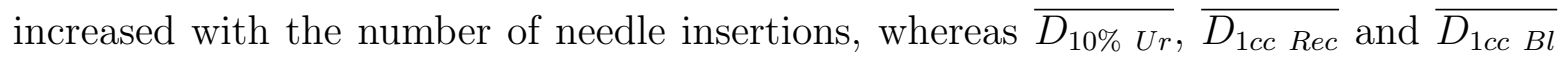
decreased. The large range between the maximum and minimum values is explained by the different anatomy of each patient especially the position of the tumor with respect to the other organs at risk. $\overline{H I_{150 \%}}, \overline{H I_{200 \%}}$ and $\overline{C O I N}$ showed the same trend as in Figures 5(a) and 5(b).

A limitation in using less needle insertions is that the robustness of the dose plan may drop. It must be kept in mind that these are only simulations: in practice, there may be errors in needle positioning or in the position of the rotation point which may lead to modifications in the dose distribution. The impact of an error in position of one needle on the dose plan must be studied in future work. Furthermore, it is important to note that in this study, no modification of the anatomy (for example due to the insertion of the needle) was taken into account. Lagerburg et al (2006) however showed that the prostate motion was significantly less when using a robotic device that taps the needle compared to hand insertion.

A way to further increase the quality of the dose plan would be to couple this method with a gradient-based optimizer on all parameters at the same time (point of rotation, needles angles and dwell times) based on the cost function described in Eq. 3. However, the dose distribution may not be drastically changed because it would only improve locally the parameters. Since on average, a clinically acceptable plan is already reached by using four needle insertions, coupling this method with a gradient-based optimization might not be necessary.

Moreover, the pipeline proposed is compatible with a re-optimization of the dose plan parameters after each insertion of the needle thanks to the proposed experimental set-up: in terms of hardware, the robotic device developed in our institution is such that the needle can be inserted needle under MRI guidance, and, in terms of software, 
the calculation time of the optimizer could be further reduced to make it eligible for intra-operative use. The development of a procedure which re-optimize of the dose plan parameters after each insertion of the needle will be also studied in future work.

Although the optimizer described here was implemented with the point source approximation for simplicity of calculation, the proposed method allows the use of more precise source models such as the line source approximation.

This optimizer workflow completes all objectives: it is developed for divergent needle patterns with a single rotation point and it optimizes the clinically relevant dose parameters of HDR brachytherapy for prostate cancer, specifically $D_{95 \% ~ P T V}, D_{10 \%}$ Ur

$D_{1 c c \text { Rec }}$ and $D_{1 c c B}$. . Unlike most optimizers such as HIPO (Karabis et al 2009) and IPSA (Hsu et al 2004), no manual determination of several input parameters as minima or maxima dose for PTV and organs at risk is necessary. Moreover, most optimizers require weight coefficients to be defined (see Dinkla et al 2014), whereas the proposed optimizer workflow does not since an automatic exhaustive search for optimal coefficient values is performed. The results prove that the optimizer workflow presented is able to obtain a clinically accepted plan with a few needle insertions already, whereas during the current clinical procedure, 13 to 17 needles are usually inserted. However, the impact of practical error in needle positioning must be studied to find the optimal number of needle insertions for real clinical procedures. Finally, the proposed optimizer took less than 20 minutes to compute although it has not been optimized in speed. Therefore, it shows great perspectives for a further reduction of the computation time by parallelizing the calculation in order to be eligible for intra-operative use.

\section{Conclusion}

In this paper, a complete inverse dose planning optimization workflow for focal-HDR prostate brachytherapy with a divergent needle pattern was presented. It can determine the optimal center of rotation, needle positioning (thus source positions) and dwell times of the sources in order to deliver the desired dose distribution for a given number of needle insertions. The optimization is made on the dose coverage (meaning the $D_{95 \% ~ P T V}, D_{10 \% \text { Ur }} D_{1 c c \text { Rec }}$ and $D_{1 c c B l}$ ) and does not need to set importance factors for the organ doses considered as input. Clinically accepted plans were obtained for on average 4 needle insertions for the 10 patients tested.

\section{Acknowledgements}

This study was funded by Philips Medical Systems Nederland B.V. The authors also thank the European Research Council (project ERC-2010-AdG-20100317, Sound Pharma). 


\section{References}

Alterovitz R, Lessard E, Pouliot J, Hsu I-C J, OBrien J F and Goldberg K 2006 Optimization of HDR brachytherapy dose distributions using linear programming with penalty costs Med. Phys. 33 4012-20.

Boyea G, Antonucci J, Wallace M, Ghilezan M, Gustafson G, Chen P Y, Saputo K, Flynn C and Martinez A 2007 Int. J. Radiat. Oncol. Biol. Phys. 69 357-8

Daskalov G M, Lffler E and Williamson J F 1998 Monte Carlo-aided dosimetry of a new high dose-rate brachytherapy source Med. Phys. 25 2200-8.

DiMaio S P, Pieper S, Chinzei K, Hata N, Haker S J, Kacher D F, Fichtinger G, Tempany C M and Kikinis R 2007 Robot-assisted needle placement in open MRI: system architecture, integration and validation Comput. Aided Surg. 12 15-24.

Dinkla A M, van der Laarse R, Kaljouw E, Pieters B R, Koedooder K, van Wieringen N and Bel A A 2014 Comparison of inverse optimization algorithms for HDR/PDR prostate brachytherapy treatment planning Brachytherapy (E-pub ahead of print).

Fischer G, DiMaio S, Iordachita I and Fichtinger G 2007 Robotic Assistant for Transperineal Prostate Interventions in 3T Closed MRI Med. Image Comput. Assist. Interv. 4791 425-33.

Fischer G S, Iordachita I, Csoma C, Tokuda J, DiMaio S P, Tempany C M, Hata N and Fichtinger G 2008 MRI-Compatible Pneumatic Robot for Transperineal Prostate Needle Placement Mechatronics 13 295-305.

Goldman S P, Turnbull D, Johnson C, Chen J Z and Battista J J 2009 Real-time fast inverse dose optimization for image guided adaptive radiation therapyenhancements to fast inverse dose optimization (FIDO) J. Appl. Phys. 105102008.

Goldman S P, Chen J Z and Battista J J 2005 Feasibility of a fast inverse dose optimization algorithm for IMRT via matrix inversion without negative beamlet intensities Med. Phys. 58 1041-57.

Gorissen B L, den Hertog D and Hoffmann A L 2013 Mixed integer programming improves comprehensibility and plan quality in inverse optimization of prostate HDR brachytherapy Phys. Med. Biol. 58 1041-57.

Groenendaal G, Moman M R, Korporaal J G, van Diest P J, van Vulpen M, Philippens M E P and van der Heide U A 2010 Validation of functional imaging with pathology for tumor delineation in the prostate Radiother. Oncol. 94 145-50.

Holm , Tedgren C, . and Larsson T 2013 Heuristics for integrated optimization of catheter positioning and dwell time distribution in prostate HDR brachytherapy Ann. Operat. Res. 1-21.

Hsu I, Lessard E, Weinberg V and Pouliot J 2004 Comparison of inverse planning simulated annealing and geometrical optimization for prostate high-dose-rate brachytherapy Brachytherapy 3 147-52.

Karabis A, Giannouli S and Baltas D 2005 HIPO: A hybrid inverse treatment planning optimization algorithm in HDR brachytherapy Radiother. Oncol. 7629.

Karabis A, Belotti P and Baltas D 2009 Optimization of Catheter Position and Dwell Time in Prostate HDR Brachytherapy using HIPO and Linear Programming World Congress on Medical Physics and Biomedical Engineering (Germany); IFMBE Proc. 25 612-5.

Kolkman-Deurloo I K K, Visser A G, Nil C G J H, Driver N and Levendag P C 1994 Optimization of interstitial volume implants Radiother. Oncol. 31 229-39.

Lessard E and Pouliot J 2001 Inverse planning anatomy-based dose optimization for HDRbrachytherapy of the prostate using fast simulated annealing algorithm and dedicated objective function Med. Phys. 28 773-79.

Lagerburg E, Moerland M A, van Vulpen M and Lagendijk 2006 J J W A new robotic needle insertion method to minimise attendant prostate motion Radiother. Oncol. 80 73-7.

Ménard C, Susil R C, Choyke P, Gustafson G S, Kammerer W, Ning H, Miller R W, Ullman K L, Sears Crouse N, Smith S, Lessard E, Pouliot J, Wright V, McVeigh E, Coleman C N and Camphausen K 2004 MRI-guided HDR prostate brachytherapy in standard 1.5T scanner Int. J. Radiat. Oncol. Biol. Phys. 59 1414-23. 
Nath R, Anderson L L, Luxton G, Weaver K A, Williamson J F and Meigooni A S 1995 Dosimetry of interstitial brachytherapy sources: Recommendations of the AAPM Radiation Therapy Committee Task Group No. 43 Med. Phys. 22 209-34.

Pieters B R, de Back D Z, Koning C C E and Zwinderman A H 2009 Comparison of three radiotherapy modalities on biochemical control and overall survival for the treatment of prostate cancer: A systematic review Radiother. Oncol. 93 168-73.

Poulin E, Fekete C C, Ltourneau M, Fenster A, Pouliot J and Beaulieu L 2013 Adaptation of the CVT algorithm for catheter optimization in high dose rate brachytherapy Med. Phys. 40 111724-9.

Polders D L, Steggerda M, van Herk M, Nichol K, Witteveen T, Moonen L,Nijkamp J and van der Heide 2015 U A Establishing implantation uncertainties for focal brachytherapy with I-125 seeds for the treatment of localized prostate cancer Acta Oncol. 1-8 (E-pub ahead of print).

Rivard M J, Coursey B M, DeWerd L A, Hanson W F, Saiful Huq M, Ibbott G S, Mitch M G, Nath R and Williamson J F 2004 Update of AAPM Task Group No. 43 Report: A revised AAPM protocol for brachytherapy dose calculations Med. Phys. 31 633-74.

Siauw T, Cunha A, Berenson D, Atamtrk A, Hsu I, Goldberg K and Pouliot J 2012 NPIP: A skew line needle configuration optimization system for HDR brachytherapy Med. Phys. 39 4339-46.

Steggerda M J, van der Poel H G and Moonen L M F 2010 Minimizing the number of implantation needles for prostate ${ }^{125} \mathrm{I}$ brachytherapy: An investigation of possibilities and implications Brachytherapy 9 319-27.

Van den Bosch M R Moman M R, van Vulpen M, Batterman J J, Duiveman E, van Schelven L J, de Leeuw H, Lagendijk J J W and Moerland M A 2010 MRI-guided robotic system for transperineal prostate interventions: proof of principle Phys. Med. Biol. 55 133-40.

Vargas C, Ghilezan M, Hollander M, Gustafson G, Korman H, Gonzales J and Martinez A 2005 A new model using number of needles and Androgen deprivation to predict chronic urinary toxicity for high or low dose rate prostate brachytherapy J. Urol. 174 882-7 